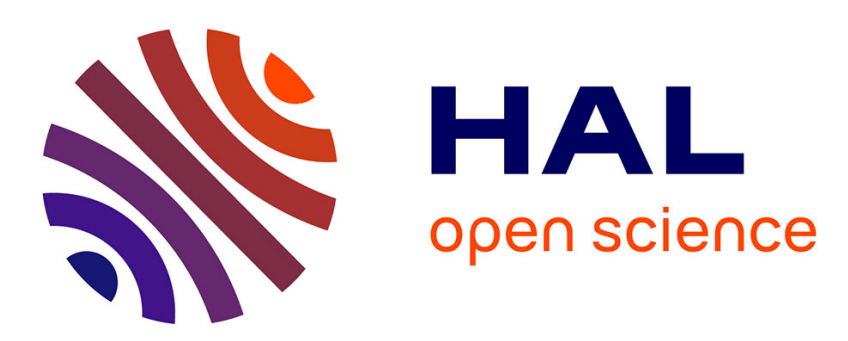

\title{
Separation of two attractive ferromagnetic ellipsoidal particles by hydrodynamic interactions under alternating magnetic field
}

\author{
Micheline Abbas, Georges Bossis
}

\section{- To cite this version:}

Micheline Abbas, Georges Bossis. Separation of two attractive ferromagnetic ellipsoidal particles by hydrodynamic interactions under alternating magnetic field. Physical Review E , 2017, 95 (6), pp.62611. 10.1103/PhysRevE.95.062611 . hal-01688236

\section{HAL Id: hal-01688236 https://hal.science/hal-01688236}

Submitted on 19 Jan 2018

HAL is a multi-disciplinary open access archive for the deposit and dissemination of scientific research documents, whether they are published or not. The documents may come from teaching and research institutions in France or abroad, or from public or private research centers.
L'archive ouverte pluridisciplinaire HAL, est destinée au dépôt et à la diffusion de documents scientifiques de niveau recherche, publiés ou non, émanant des établissements d'enseignement et de recherche français ou étrangers, des laboratoires publics ou privés. 


\title{
Separation of two attractive ferromagnetic ellipsoidal particles by hydrodynamic interactions under alternating magnetic field
}

\author{
Micheline Abbas* \\ Laboratoire de Génie Chimique, Université de Toulouse, CNRS, INPT, UPS, Toulouse, France \\ Georges Bossis \\ Laboratoire de Physique de la Matière Condensée, Nice, France \\ (Received 20 February 2017; revised manuscript received 30 May 2017; published 29 June 2017)
}

\begin{abstract}
In applications where magnetic particles are used to detect and dose targeted molecules, it is of major importance to prevent particle clustering and aggregation during the capture stage in order to maximize the capture rate. Elongated ferromagnetic particles can be more interesting than spherical ones due to their large magnetic moment, which facilitates their separation by magnets or the detection by optical measurement of their orientation relaxation time. Under alternating magnetic field, the rotational dynamics of elongated ferromagnetic particles results from the balance between magnetic torque that tends to align the particle axis with the field direction and viscous torque. As for their translational motion, it results from a competition between direct magnetic particle-particle interactions and solvent-flow-mediated hydrodynamic interactions. Due to particle anisotropy, this may lead to intricate translation-rotation couplings. Using numerical simulations and theoretical modeling of the system, we show that two ellipsoidal magnetic particles, initially in a head-to-tail attractive configuration resulting from their remnant magnetization, can repel each other due to hydrodynamic interactions when alternating field is operated. The separation takes place in a range of low frequencies $f_{c 1}<f<f_{c 2}$. The upper frequency limit $f_{c 2} \tau_{r} \approx 0.04$ (where $\tau_{r}$ is the rotation time scale) depends weakly on the ratio of magnetic field to particle magnetization strength, whereas $f_{c 1}$ tends to zero when this ratio increases.
\end{abstract}

DOI: 10.1103/PhysRevE.95.062611

\section{INTRODUCTION}

Magnetic particles have the great advantage of being tunable by external magnetic field. Under uniform static magnetic field, they are known to form fibrous structures that change the bulk rheological properties of the suspending fluid. These suspensions, typically referred to as magnetorheological fluids (MR) [1], have been employed in a variety of damping and shock absorbing devices, and the same principle applies to spherical and elongated elementary particles [2,3].

In biomedecine, magnetic nanoparticles can be used in experimental cancer treatment called magnetic hyperthermiaan increase in temperature resulting from the thermal energy released by magnetic nanocrystals in an external alternating magnetic field [4]. Also magnetic nanoparticles can be conjugated with carbohydrates to detect bacteria. Iron oxide particles have been used for the detection of Gram-negative bacteria like Escherichia coli and for detection of Grampositive bacteria like Streptococcus suis [5]. In these emergent applications, it can be of interest to concentrate or detect molecules in vitro by adsorbing them on the particle surface. Nanoparticles have large specific surface area allowing them to capture biological molecules. Although in the most general case the particles tend to be quasispherical, there is an interest in elongated particles due to their greater magnetization capacity compared to spherical ones (the demagnetization tends towards zero when a particle approaches a needle), which facilitates their detection by magnets. If they are functionalized with biological molecules, the particle response to a magnetic field is a very practical way to dose the adsorbed

\footnotetext{
*micheline.abbas@ensiacet.fr
}

matter. Also, elongated particles induce measurable optical anisotropy. This anisotropy is used, for instance, to detect the presence of hemozoin crystals, an ellipsoidal magnetic particle synthesized during the destruction of hemoglobin cells by malaria [6].

When compared to isotropic ones, anisotropic particles can be easily torqued by an external field, and the corresponding orientation can be visualized by simple optical techniques [7]. The torque features are attractive to several applications, where anisotropic magnetic particles are used as force sensors, microstirrers, active components in constrained geometries, microrheological probes [8,9], or externally actuated micropropellers [10,11]. One can benefit from the very rich orientation dynamics of an assembly of magnetic particles submitted to an oscillating magnetic field. As an example, Ref. [12] formed colloidal ribbons self-assembled from microscopic magnetic ellipsoids of permanent magnetic moment perpendicular to their long axis. The assembly performed using static magnetic field was reoriented when the external applied field oscillates.

While the formation of assemblies is the basis of the multiple applications cited above, particle clustering can be undesired typically in optical applications. Therefore, in this paper, we are interested in exploring a plausible way of separating a couple of attractive magnetic particles, thanks to the torque applied by an external magnetic field. When elongated magnetic particles are submitted to alternating magnetic field, the system is subject to a competition between direct magnetic particle-particle interactions and solvent-flow-mediated hydrodynamic interactions (HI), leading to translation-rotation couplings that are more intricate than the coupling found for spherical particles studied in Ref. [13]. 
We consider for this study the case of elongated ferromagnetic microparticles. Elongated particles like cobalt with a size $O(100 \mathrm{~nm})$ can be in the single domain state, which allows them to have remnant magnetization even in the absence of the magnetic field, without being subject to Brownian motion: the dipolar coupling parameter, which is the ratio of the energy of dipole-dipole interactions and the particle thermal energy, is relatively high, $O(1000)$. Particles are modeled as ellipsoids and are considered to have remnant magnetization, as if they have been exposed to a magnetic field prior to the application of an alternating field, and Brownian motion is not taken into account (the particle size is close to a micron). In cases where thermal fluctuations cannot be neglected (nanosized particles), their impact on particle motion is diffusive, which favors particle separation in dilute systems. A dipolar representation is used to account for pairwise magnetic interactions [14]. In the case of one particle pair, the dipolar interaction is sufficient to capture the primary magnetic interaction, unlike concentrated systems, where detailed multipole representation may be warranted to resolve very localized variations in the magnetic field.

The paper is organized as following. In Sec. II, we explain the model and show the results for the translation and rotation trajectories of a pair of particles with no HI. Section III shows the impact of $\mathrm{HI}$ on the relative trajectories as a function of the alternating field frequency. Stokesian hydrodynamic interactions are taken into account in the frame of the forcecoupling method. We focus especially on the case of a pair of particles in a head-to-tail initial configuration. A simple model based on Stokes fundamental solutions is used to explain the impact of the frequency on the particle pair separation. This section also includes a discussion on the effect of varying separately particle magnetization-to-magnetic field ratio, as well as the particle aspect ratio. The paper is ended with conclusive remarks.

\section{SYSTEM DYNAMICS IN THE ABSENCE OF HI}

This section sets the problem and the relevant parameters that come into play. A cluster here consists of two elongated particles that tend to aggregate if they are submitted to a constant magnetic field. A theoretical model for the dynamics of the particle pair is written and solved, first with neglected HI between moving particles. Elongated particles, called here fibers, are modeled as prolate spheroids with minor semiaxis $a$ and major semiaxis $\lambda a$. The aspect ratio $\lambda$ of the so-called fiber is larger than unity, and it is taken equal to 6 for all the results shown in this paper.

\section{A. Single fiber}

The fibers are assumed to have constant magnetic dipole intensity (remnant magnetization), with $\mathbf{M}$ oriented along the fiber axis. Its dipole moment is therefore $\mathbf{m}=\mu_{0} V \mathbf{M}$, where $\mu_{0}$ is the vacuum magnetic permeability $\left(\mu_{0}=4 \pi 10^{-7}\right.$ in $H / m$ or $\left.N / A^{2}\right)$ and $V$ is the particle volume $\left(V=\frac{4}{3} \pi \lambda a^{3}\right.$ for spheroid).

Under alternating magnetic field $H=H_{0} \cos (\omega t) \mathbf{e}_{y}$, where $\omega=2 \pi f$ and $f$ is the frequency of the magnetic field, the fiber is submitted to a magnetic torque $\mathbf{T}_{m}=\mathbf{m} \times \mathbf{H}$. If $\theta$ is
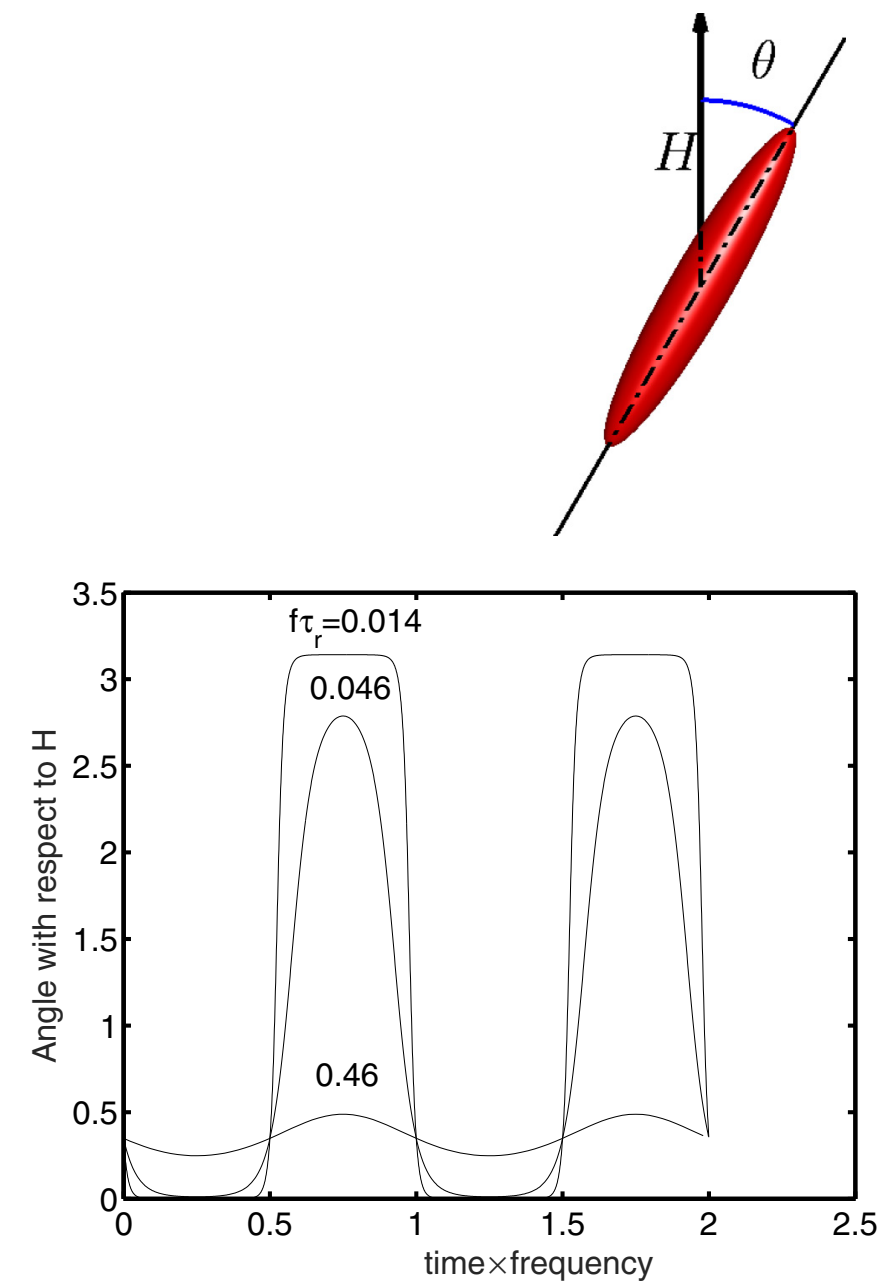

FIG. 1. Top panel: sketch of the fiber under magnetic field. Bottom panel: angular motion of one particle under alternating magnetic field for different frequencies: $f \tau_{r}=0.014,0.046$, and 0.46 .

the angle between the field direction and the fiber principal axis (following Fig. 1), then the magnetic torque intensity is $\mathbf{T}_{m}=-\beta \cos (\omega t) \sin \theta$, with $\beta=\mu_{0} V M H_{0}$.

In a viscous fluid, the fiber also experiences hydrodynamic torque from viscous friction. In the absence of hydrodynamic interactions between particles, the viscous torque is linearly related to the speed of rotation of the fiber through $T_{h}=-\alpha \dot{\theta}$. The hydrodynamic coefficient $\alpha$ is proportional to the fluid viscosity and depends on the fiber aspect ratio (the dot refers to the derivative in time) and on the Reynolds number based on the fiber rotational motion. The present study is performed in the limit of negligible fluid inertia. This means that for a fiber of length $L$ rotating at angular frequency $\omega$ in a fluid of density $\rho_{f}$ and viscosity $\mu$, the Reynolds number $\operatorname{Re}=\rho_{f} L^{2} \omega / \mu_{f}$ is very small. For a fiber of length $1 \mu \mathrm{m}$ in water, the case $\mathrm{Re} \ll 1$ corresponds to a rotation speed $\omega \ll 10^{6} \mathrm{rad} /$ s or $f \ll$ $160 \mathrm{kHz}$. In this limit, the microhydrodynamics principles fully apply. The friction coefficient of a prolate spheroid rotating around one of the small axis can be modeled following Ref. [15] (also written in Ref. [16]):

$$
\alpha=\frac{16}{3} \pi \mu a^{3} \delta^{3}\left(1+\lambda^{2}\right)\left[\left(2 \delta^{2}+1\right) \log (\lambda+\delta)-\lambda \delta\right]^{-1},
$$


TABLE I. Typical system parameters.

\begin{tabular}{lc}
\hline \hline Liquid phase & Water \\
\hline Fiber length & $1 \mu \mathrm{m}$ \\
Fiber aspect ratio & $\lambda=6$ \\
Fiber material & Cobalt \\
Magnetic field intensity & $H_{0}=5 \mathrm{kA} / \mathrm{m}$ \\
Particle magnetization & $M=5 \mathrm{kA} / \mathrm{m}$ \\
Fiber rotation time scale & $\tau_{r}=1 \mathrm{~ms}$ \\
\hline \hline
\end{tabular}

with $\delta^{2}=\left|\lambda^{2}-1\right|$. If particle inertia is negligible, meaning that the time relaxation due to change in particle position and orientation is very small compared to the particle rotation time scale $\left(\rho_{p} a^{2} / \mu \ll 1\right)$, the sum of the torques that a particle experiences cancels; i.e., $\mathbf{T}_{m}+\mathbf{T}_{h}=0$. The equation of angular motion becomes $[-\alpha \dot{\theta}-\beta \cos (\omega t) \sin \theta=0]$, and the corresponding solution starting from the initial angle $\theta_{0}$ satisfies

$$
\theta(t)=2 a \tan \left[-\tan \left(\frac{\theta_{0}}{2}\right) e^{-\frac{1}{\omega \tau_{r}} \sin \omega t}\right] .
$$

We define the rotation time scale $\tau_{r}$ from the ratio $\alpha / \beta$ that appears in the angular motion equation:

$$
\tau_{r}=\frac{\alpha}{\beta}=\frac{4 \mu}{\mu_{0} H_{0} M} f(\lambda) .
$$

Note that the rotation time does not depend on the particle size but on the aspect ratio via the function $f(\lambda)=$ $\delta^{3}\left(1+\lambda^{2}\right)$ $(\approx 9.09$ for $\lambda=6) \cdot \tau_{r}$ is the characteristic time scale in the considered problem. Typical parameters that describe the system are summarized in Table I.

The angular motion of one particle submitted to the magnetic field oscillation is displayed in Fig. 1. The maximum angle that the particle can reach with respect to the initial magnetic field direction $\mathbf{H}_{0}$ depends on the oscillation frequency scaled by the rotation time scale. At low frequency, the particle becomes perfectly aligned with the magnetic field direction and the relative orientation with respect to the field alternates between 0 and $\pi$. This is called the oscillation regime. At high frequency, the particle major axis vibrates around an average value, failing to align with the magnetic field direction. This is the vibration regime.

\section{B. Two fibers}

Ferromagnetic particles experience magnetic dipolar forces. By analogy with electrostatics, the induced dipole moment of each elongated particle is supposed to be a result of two opposite point charges $q$ and $-q$ near the particle tips. Following Bossis et al. [17], the charge value $q$ is related to the fiber dipole moment $m=q L$. The charges are placed at a distance $a$ from each fiber extremity (see the sketch on the top-left corner of Fig. 2). The magnetic interparticle forces are reduced to forces between charges written as

$$
\mathbf{F}_{m}= \pm \frac{q^{2}}{4 \pi \mu_{0}} \frac{\mathbf{r}^{*}}{r^{* 3}}= \pm\left[\mu_{0} M^{2} a^{2}\right] \frac{\pi}{9} \frac{\mathbf{r}^{*}}{r^{* 3}},
$$

where $\mathbf{r}^{*}$ is the distance between charges scaled with $a$, and $\mu_{0} M^{2} a^{2}$ is the force characteristic scale.
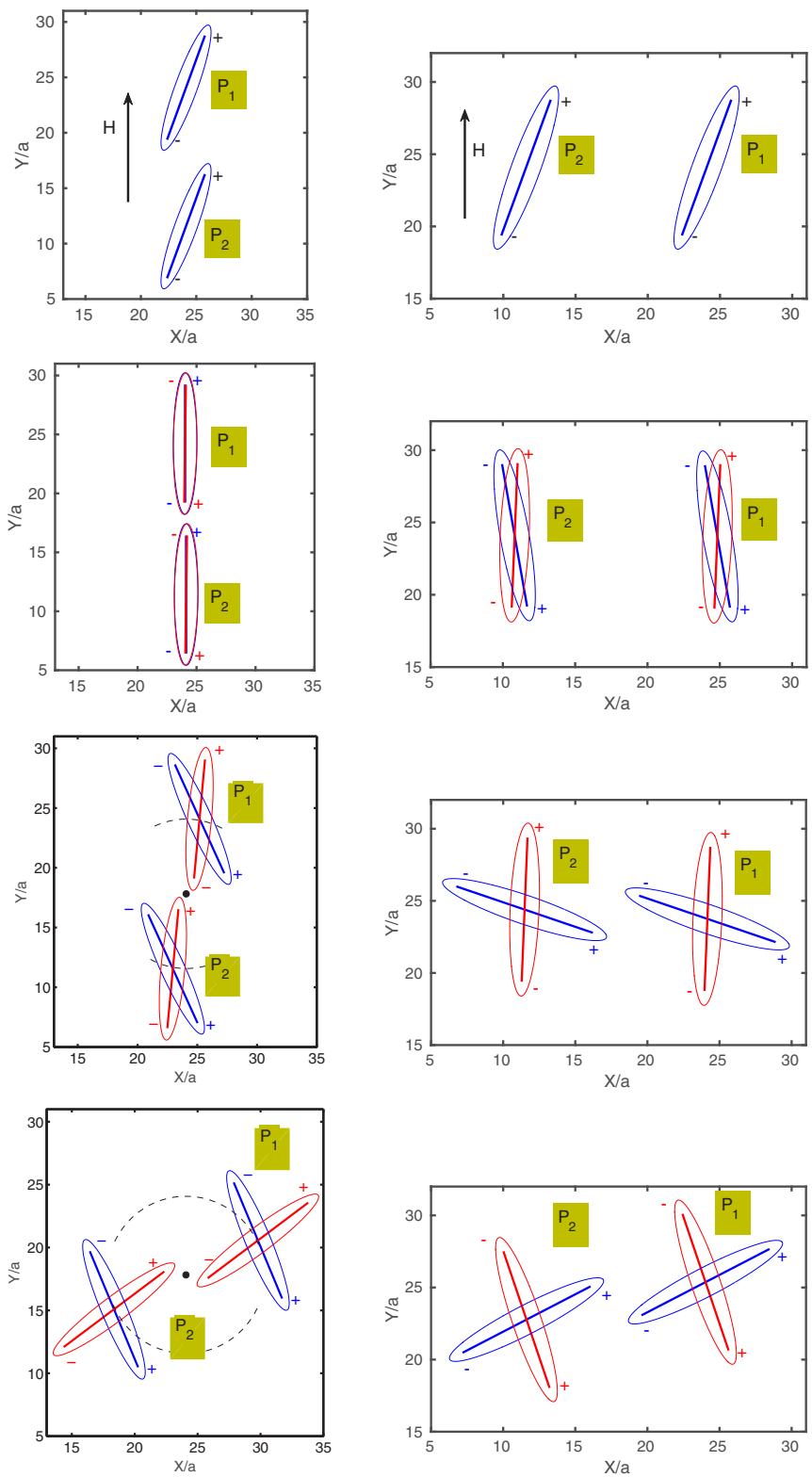

FIG. 2. Effect of the frequency on the behavior of two fibers under alternating magnetic field in the absence of HI. Left (case A) and right (case B) columns correspond to different initial configurations with the line joining fiber centers aligned with (and, respectively, perpendicular to) the magnetic field direction. The + and - charges are placed at the extremity of the continuous lines following the ellipsoid major axis. The magnetic moment of every particle is oriented from - to + . The first row corresponds to the initial state of the system. The second to fourth rows correspond to dimensionless frequencies $f \tau_{r}=0.023,0.069$, and 0.138 , respectively, with $H M=10^{10}$ and $H / M=1$. In these rows, blue and red colors correspond to the system configuration at chosen time $t$ and $t+1 /(2 f)$, respectively. The circular arcs indicate the relative trajectory of the center of $P_{2}$ with respect to $P_{1}$ in time.

A repulsive force must be included to avoid particles from overlapping, both here and in the next section, where HI will be taken into account without fully resolving lubrication near particle contact. The force used here 
is classical in computer simulations of the dynamics of magnetic particles:

$$
\mathbf{F}_{r}=\left[\mu_{0} M^{2} a^{2}\right] e^{-\xi\left(\left|r^{*}\right|-2.2\right)} \frac{\mathbf{r}^{*}}{r^{*}} .
$$

The parameter $\xi$ is set to 10 so that for two particles that are in mechanical contact the repulsive force exactly balances the attractive magnetic interaction [18] when the force is applied at the position of charges. The sum of dipolar and repulsive forces constitutes the interparticle forces $\mathbf{F}_{P_{1} P_{2}}=-\mathbf{F}_{P_{2} P_{1}}$. The notation $\mathbf{F}_{P_{1} P_{2}}$ refers to the force applied at particle $P_{1}$ from interaction with particle $P_{2}$. The point of application of interparticle forces is located near the particle extremities, leading to additional torques, noted as $\mathbf{T}_{P_{1} P_{2}}$ and $\mathbf{T}_{P_{2} P_{1}}$ at particles $P_{1}$ and $P_{2}$, respectively.

The third force is the viscous drag. A particle, which center of mass has a translational velocity $\dot{\mathbf{Y}}_{P}$, experiences viscous drag force $\mathbf{F}_{h}$ opposite to the direction of motion. Assuming hydrodynamic interactions are negligible, the drag force expression is identical to the isolated particle case; i.e., $\mathbf{F}_{h}=-\mathbf{K} \cdot \dot{\mathbf{Y}}_{P}$. The resistance matrix $\mathbf{K}$ is diagonal in the principal frame of a symmetrical particle, and its coefficients depend on the particle shape. For a spherical particle of radius $a, \mathbf{K}=6 \pi \mu a \mathbf{I}$, where $\mathbf{I}$ is the identity tensor. For a prolate spheroid of major axis direction 1 and minor axis directions 2 and $3, K_{11}=8 \pi \mu a \delta^{3}\left[\left(2 \delta^{2}+1\right) \log (\lambda+\delta)-\lambda \delta\right]^{-1}$ and $K_{22}=K_{33}=16 \pi \mu a \delta^{3}\left[\left(2 \delta^{2}-1\right) \log (\lambda+\delta)-\lambda \delta\right]^{-1} \quad$ (from Ref. [15] written in Ref. [16]). The translation equation of motion is therefore $\mathbf{F}_{h}+\mathbf{F}_{m}+\mathbf{F}_{r}=0$.

If we neglect hydrodynamic interactions, the viscous torque applied to a rotating particle is $\mathbf{T}_{h}$ identical to the expression of the single particle given in the previous subsection. The two-body problem is restricted to one plane containing the magnetic field and the relative particle positions. In this plane, the equations for rotational and translational motion of particle $P_{i}\left(P_{j}\right.$ being the other particle) are given by

$$
\begin{gathered}
\dot{\theta}_{P_{i}}=\frac{1}{\alpha}\left(\mathbf{T}_{P_{i} P_{j}}+\mathbf{T}_{m, P_{i}}\right), \\
\dot{\mathbf{Y}}_{P_{i}}=\mathbf{K}^{-1} \cdot \mathbf{F}_{P_{i} P_{j}} .
\end{gathered}
$$

The time integration of the particle angular and translational motion was done using Adams Bashforth scheme of order 3, with a time step $d t \approx \tau_{r} / 60$. The behavior of the system without hydrodynamic interactions discussed in this section is globally not dependent on the time step, neither on the strength of the repulsive force (parameter $\xi$ ).

We consider two configurations where the initial distance between particle centers is $r_{0}=12.5 a$, and the initial angle of orientation of both fibers $\theta_{0}=20^{\circ}$. In configuration A (left column of Fig. 2), the line joining particle centers is initially parallel to the magnetic field direction. In configuration $\mathrm{B}$ (right column), the line of centers is perpendicular to the magnetic field. If the magnetic field was invariant in time, the particles would first rotate toward the field direction, then get aggregated in case A (in a head-to-tail configuration) and repelled in case B. Under alternating magnetic field, we follow the evolution of angular and relative particle distance in the directions normal and parallel to the magnetic field using $\Delta X=X_{P 1}-X_{P 2}$ and $\Delta Y=Y_{P 1}-Y_{P 2}$, respectively. The relative trajectories are displayed in Fig. 3. Two behaviors are observed:

In case $\mathrm{B}$, at low frequency, particles tend to separate along the $x$ direction (see the monotonous increase of the absolute value of $\Delta X$ and $\Delta Y$ ). The system conserves globally its repulsive nature earned from the initial configuration. However, when the frequency increases, the distance between particle centers remains almost constant in time, meaning that at high angular oscillation the average in time of the interparticle force tends to zero.

In case $\mathrm{A}$, we distinguish three regimes. At low frequency, the angle $\theta$ of both particles oscillates under the alternating magnetic field. The line of centers remains parallel to the magnetic field. At intermediate frequencies, the angular orientation of both particles oscillates between $-\pi$ and $\pi$. At long times, the relative trajectories of their centers can be described by circular arcs of radius $\lambda a$ (as sketched in Fig. 2). This corresponds to a large-scale oscillation of the relative positions $\Delta X$ and $\Delta Y$. At high frequencies, the line of centers becomes perpendicular to the magnetic field direction at long time $(\Delta X \rightarrow 2 \lambda a$ and $\Delta Y \rightarrow 0$, according to Fig. 3).

Note that the problem of angular motion of a pair of ellipsoidal particles is to some extent similar to the problem studied by Ref. [19] relative to the synchronization of spherical particle oscillations in an AC field due to dipolar interactions. It would have been of interest to consider two time scales, a short one related to the period of the AC field and a longer one related to the evolution of the amplitude of the oscillations, so that a stability analysis could be done like in Ref. [19]. However, holding the particle center of gravity fixed, completely changes their rotational dynamics, so this analysis is not possible without taking into account the coupling with the translational equations, which would be quite difficult to realize. Instead, we have studied the rotational dynamics by the direct integration of the set of equations over a time long enough to distinguish between different dynamics and so to delimit different regimes depending on the frequency of the applied field. The summary on the dynamics of the particle pair in case $\mathrm{A}$ is the following. $A 1$ synchronous continuous rotation at low frequency, $A 2$ asynchronous oscillation of $\pi$ at intermediate frequency (as shown in Fig. 4), and $A 3$, oscillation (of an angle less than $\pi$ ) at high frequency. For example, we observe an asynchronous rotation, in the range $f \tau_{r} \approx[0.017-0.04]$ for case A with $H_{0} / M=1$. Note that the intermediate regime depends on the initial particle orientation with respect to the magnetic field. The first two regimes do not lead to a significant change of the initial configuration because particles spend a consequent time while they keep aligned, which increases the attraction between opposite charges. The third regime leads to the change in the particle configuration where the particle center line becomes perpendicular to the magnetic field since oscillating particles spend less time aligned with the field where the attractive magnetic interactions are the most important. In all cases, the particle distance remains constant. 

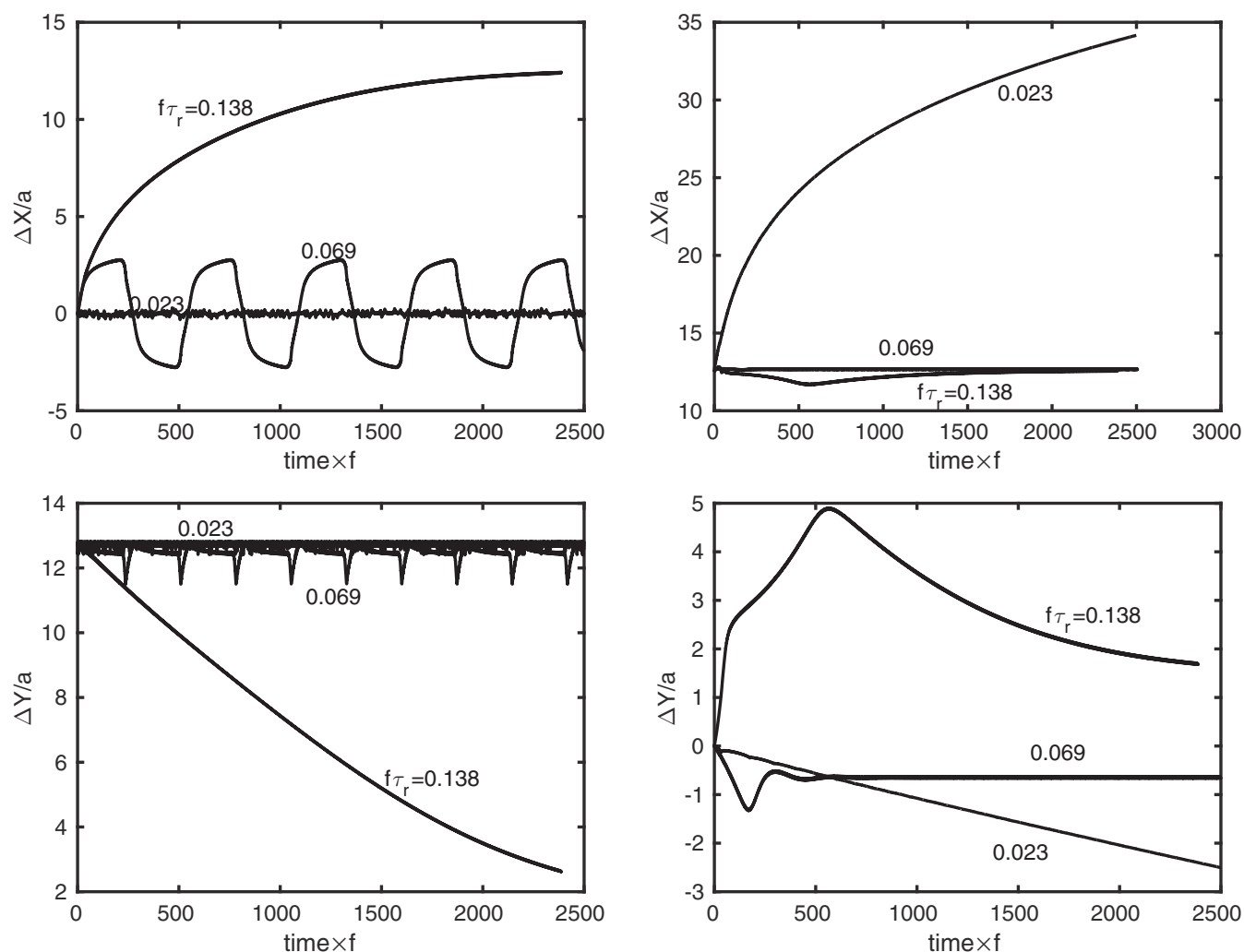

FIG. 3. Evolution of particle relative position in time in the absence of $\mathrm{HI}$ for different frequencies $f \tau_{r}=0.023,0.069$, and 0.138 , and for $H / M=1$. First row: $\Delta X=x_{P 1}-x_{P 2}$. Second row: $\Delta Y=y_{P 1}-y_{P 2}$. Left column, case A; right column, case B.

\section{EFFECT OF HYDRODYNAMIC INTERACTIONS}

\section{A. Numerical simulations based on the force-coupling method (FCM)}

Particle-resolved direct numerical simulations were performed to calculate the hydrodynamic interactions between the particles submitted to the forces and torques described above. The numerical simulation of particle trajectories and suspension flow field is based on the force-coupling method (called

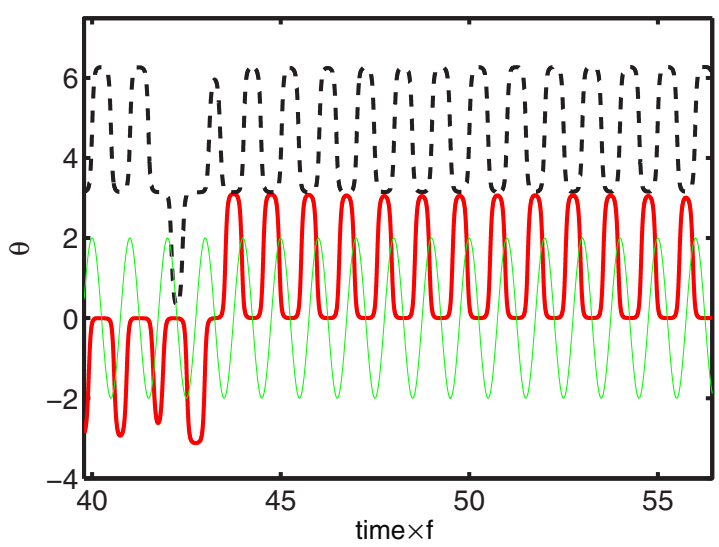

FIG. 4. Asynchronous flip-flop motion of particles observed from the angular trajectories of both particles (red and black) without hydrodynamic interactions, for $H_{0} / M=1$ and $f \tau_{r}=0.032$. Initial configuration follows case A with $\theta_{10}=\pi / 9$ and $\theta_{20}=\pi / 3$. The green line is the $2 \cos (\omega t)$. here FCM). The method initially developed for spherical particles, as described in Refs. [20] and [21], was then adapted to ellipsoidal particles by Ref. [16] (see their paper for careful description and validation of the numerical method). Flow equations (here Stokes equations) are dynamically coupled to Lagrangian tracking of particles. The fluid is assumed to fill the entire simulation domain, including the volume occupied by the particles. The fluid velocity and pressure fields are solutions of the mass and momentum conservation equations. The presence of a particle in the fluid is then represented by a body force distribution written as a multipole expansion in the Navier-Stokes equations. Only the first two terms of the expansion are deployed. The first term of the expansion, called the monopole, represents the force that the particle exerts on the fluid (due to magnetic forcing and particle-to-particle repulsive forces). The second term, called dipole, consists of an antisymmetric part related to the magnetic and interparticle torques that a particle applies on the fluid and a symmetric part that ensures that the strain rate within the fluid volume occupied by the particle is zero (particles are solid bodies). The distribution of particle forcing on the Eulerian grid (used to solve the flow equations) is done thanks to anisotropic Gaussian functions that take into account the ellipsoidal shape of the particle. The particle translational (respectively, rotational) velocities is obtained from a local weighted average of the volumetric fluid translational (respectively, rotational) velocity field over the region occupied by the particle. The trajectory of every particle is then obtained from numerical integration of its equation of motion. The angular motion of one particle obtained from numerical simulations is compared 

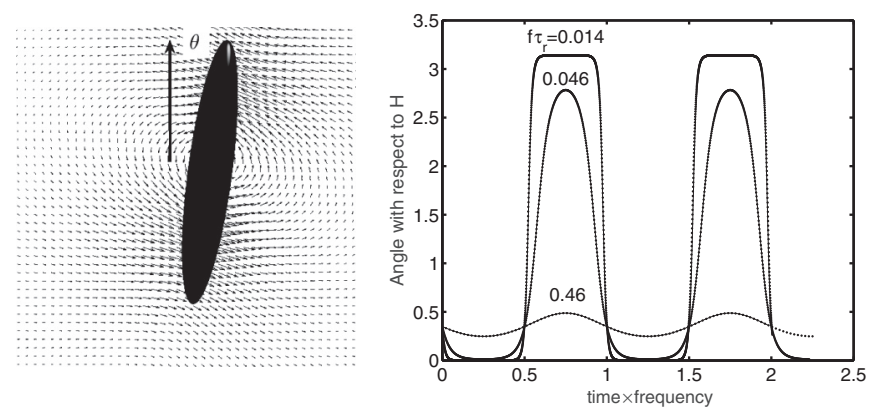

FIG. 5. Sketch of the fiber under magnetic field on the left panel with the velocity perturbation in the fluid induced by the particle rotation. On the right, angular motion of one particle under alternating magnetic field for different frequencies $f \tau_{r}=0.014,0.046$, and 0.46 : comparison of FCM results (dotted lines) with the theoretical solution (solid lines). Solid and dashed lines cannot be distinguished due to the good agreement.

to the analytical solution in Fig. 5. The excellent agreement shows that this method is accurate, both in calculating the angular motion of the particle under an external torque and in detecting the instant of particle departure from one equilibrium orientation to the other.

\section{B. Particle separation depends on the field frequency}

Simulations based on the FCM were performed starting from both configurations $\mathrm{A}$ and $\mathrm{B}$, as in the previous section. The relative particle trajectories in configuration $\mathrm{B}$ are not significantly influenced by HI. At frequency up to $f \tau_{r}=0.046$, the fibers exhibit separation along the direction perpendicular to the magnetic field as sketched in Fig. 7. At higher frequencies, the sum of applied forces tend to zero when averaged in time, and the particle relative distance remains constant. Nevertheless in case A, the impact of alternating field on fiber separation is drastically changed when HI are accounted for. The system becomes repulsive within a range of low frequencies $\left(f \tau_{r}=[0.0037-0.023]\right.$ when $\left.H_{0}=M\right)$.
Initial attractive state

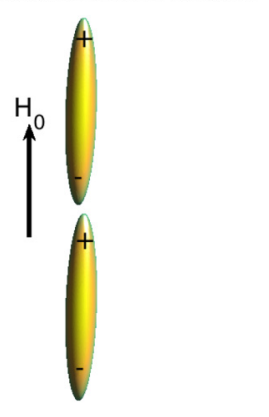

FIG. 7. Left panel: initial state of two ferromagnetic elongated particles. Right panel: position and orientation of both particles after 3.5 period of oscillation of the magnetic field, revealing the separation of these particles due to hydrodynamic interactions. The charges represent the magnetic dipole orientation of every particle: opposite and equal charge signs induce particle-particle attraction and repulsion, respectively.

As a matter of fact, for the system of ferromagnetic fibers of length $1 \mu \mathrm{m}$ in water, the corresponding frequency range is [25-250] Hz using $H=M=5 \mathrm{kA} / \mathrm{m}$, which remains in the Stokesian flow domain.

The relative trajectories in $x$ and $y$ directions of particles initially placed according to configuration A are displayed in Fig. 6, at different frequencies. While they exhibit oscillatory motion under the alternating field, particles repel each other. Their relative motion can be divided in two stages.

(1) In a first stage, the lower particle is lifted upward (the upper particle is pushed downward) with respect to the magnetic field direction, until the line between both centers becomes almost perpendicular to the magnetic field $(\Delta Y \approx 0)$. The duration of the first stage is quite short, since it takes 5 to 10 periods of magnetic field oscillation so that this lift-up process is completed. It is probably not striking that separation happens when HI are taken into account because velocity perturbations induced by particle motion are of repulsive nature. However,
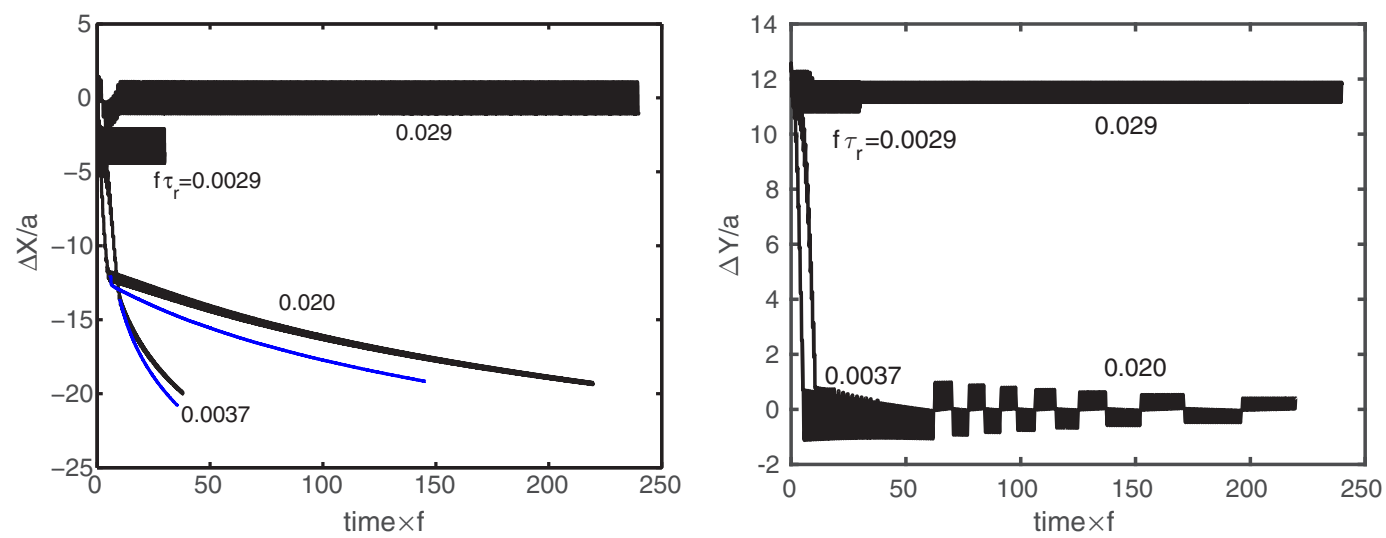

FIG. 6. Evolution of particle relative position in time (starting from configuration A) for different frequencies and $H / M=1$. The four black lines are from numerical simulations taking into account $\mathrm{HI}$ for four different frequencies. The two blue lines represent particle trajectories in the second stage (in the $x$ direction), which would be obtained without HI for the two frequencies $f \tau_{r}=0.0037$ and 0.02 . The large thickness of the black lines are due to fluctuations in particle relative positions while oscillating under the alternating magnetic field. 
what is totally unexpected is that the transition from the aggregated to repulsive state, when the frequency is increased, is very sharp. At $f \tau_{r}=0.0029$ the system remains aggregated, whereas separation happens at $f \tau_{r}=0.0037$.

(2) In a second stage, repulsive motion occurs exclusively in the $x$ direction and net separation happens. Note that, in this second stage, the repulsive motion in the $x$ direction is slower than in the first one. A net slope reduction can be noticed on $\Delta X$ curves. Some observations are noted on the particle trajectories during this second stage. The first one is that the repulsive motion in the $x$ direction is not due to the same repelling mechanism as in configuration B without HI. In the latter case, particles are subject to a drift due to magnetic interparticle forces (between identical charges) exclusively. The relative motion in the $x$ direction, calculated once the first stage is completed and with switching $\mathrm{HI}$ off, is displayed in Fig. 6 with blue lines. When HI are accounted for, the rotation of one particle exerts a hydrodynamic translation force on the other particle due to shape anisotropy, via the torque-velocity coupling, in addition to the repulsion due to magnetic forces. The separation process is slowed down by HI. The second observation is that particle rotation takes place in the same direction during the first stage, whereas particles can alternate their direction of rotation during the second stage. The magnetic field direction is a kind of vertical barrier that an isolated particle cannot cross when oscillating exclusively under magnetic torque. Crossing this barrier is made possible thanks to the additional torque coming from inter-particle forces, and the direction of rotation in time is essentially monitored by the sign of these torques.

Note that the results shown here are obtained starting from configuration A as an example. In experiments, aggregated needles often follow tail-to-head configuration with one particle among the pair inclined of a small angle $\left(5^{\circ}\right.$ for instance) with respect to the magnetic field direction. This is due to defaults on particle surfaces. We realized some tests (not shown here) starting with such configuration. They obey to the same dynamics as case A with respect to the frequency.

\section{Comparison with a simplified HI model}

In order to understand the mechanism responsible of cluster breakup by HI, we reconsidered the equations of motion, Eqs. (6) and (7), and supplemented them by an estimation of the velocity perturbations induced on each particle from the translational and rotational motion of the other one. Knowing that the source of motion of each particle is due to magnetic torque and dipolar force interactions, the velocity perturbation is estimated by using a linear distribution of point forces and point torques along the fiber axis. For a simple explanation of point force and torque solutions, see Ref. [22]. The prediction of linear and rotational velocities from the slender-body theory, first introduced by Batchelor [23], could be more accurate for these elongated particles. However, we show a posteriori that the simple model used here is enough to capture the particle relative trajectories and their dependence on the frequency.
We define $\omega_{12}$ and $\mathbf{u}_{12}$ as the total rotational and translational velocity perturbations induced at $P_{1}$ from the imposed forces and torques applied at particle $P_{2} . \omega_{12}$ is $O\left(a^{3} / r^{3}\right)$ less than the angular velocity induced by the magnetic torque and can therefore be neglected. The perturbation velocity $\mathbf{u}_{12}$ at particle $P_{1}$ resulting from the translation and rotation of particle $P_{2}$ can be calculated, using the combination of point force and point torque solutions:

$$
\mathbf{u}_{12}=\mathbf{u}_{S @ P_{1}}+\mathbf{u}_{R @ P_{1}} .
$$

The velocity perturbation $\mathbf{u}_{S}$ at particle $P_{1}$ generated by the force $\mathbf{F}_{P 2 P 1}$ (applied at particle $P_{2}$ ) is called Stokeslet in the literature on microhydrodynamics. At a position $\mathbf{x}$, the Stokeslet velocity perturbation from the force applied at $\mathbf{x}_{P 2}$ is modeled as $\mathbf{u}_{S}\left(\mathbf{x}-\mathbf{x}_{P 2}\right)=\mathbf{G}\left(\mathbf{x}-\mathbf{x}_{\mathbf{P 2}}\right) \cdot \frac{\mathbf{F}_{P 2 P 1}}{8 \pi \mu}$, using the Oseen-Burger tensor $\mathbf{G}(\mathbf{x})=\left(\frac{\mathbf{I}}{r}+\frac{\mathbf{x x}}{r^{3}}\right)$. The velocity perturbation $\mathbf{u}_{R}$ at particle $P_{1}$ generated by the torque $\mathbf{T}_{P_{2}}=\mathbf{T}_{P_{2} P_{1}}+\mathbf{T}_{m, P_{2}}$ is called Rotlet and is written as $\mathbf{u}_{R}\left(\mathbf{x}-\mathbf{x}_{P 2}\right)=\frac{\mathbf{T}_{P 2}}{8 \pi \mu} \times \mathbf{R}\left(\mathbf{x}-\mathbf{x}_{\mathbf{P 2}}\right)$, using the Rotlet tensor $\mathbf{R}(\mathbf{x})=\frac{\mathbf{x}}{r^{3}}$. Considering a uniform linear distribution of the point forces and torques along the major axis of $P_{2}$, the perturbation velocity at $P_{1}$ can be written as

$$
\begin{aligned}
\mathbf{u}_{S @ P_{1}} & =\frac{1}{L^{2}} \int_{0}^{L} \int_{0}^{L} \mathbf{u}_{S}\left(\mathbf{x}_{1}-\mathbf{x}_{2}\right) d x_{1} d x_{2} \\
& =\frac{1}{8 \pi \mu} \frac{1}{L^{2}} \int_{0}^{L} \int_{0}^{L} \mathbf{G}\left(\mathbf{x}_{1}-\mathbf{x}_{2}\right) \cdot \mathbf{F}_{P_{2} P_{1}} d x_{1} d x_{2}, \\
\mathbf{u}_{R @ P_{1}} & =\frac{1}{L^{2}} \int_{0}^{L} \int_{0}^{L} \mathbf{u}_{R}\left(\mathbf{x}_{1}-\mathbf{x}_{2}\right) d x_{1} d x_{2} \\
& =\frac{1}{8 \pi \mu} \frac{1}{L^{2}} \int_{0}^{L} \int_{0}^{L} \mathbf{T}_{P_{2}} \times \mathbf{R}\left(\mathbf{x}_{1}-\mathbf{x}_{2}\right) d x_{1} d x_{2} .
\end{aligned}
$$

Finally, note that the perturbation velocity at $P_{2}$ is $\mathbf{u}_{21}=$ $-\mathbf{u}_{12}$, because the forces applied on both particles are equal and opposite, the torques are equal and the coefficients of matrices $\mathbf{G}$ and $\mathbf{R}$ are even and odd functions, respectively.

The set of translation and rotation equations (being similar for both particles, it is only written for $P_{1}$ ) becomes

$$
\begin{gathered}
\dot{\theta}_{P_{1}}=\frac{1}{\alpha}\left(\mathbf{T}_{P_{1} P_{2}}+\mathbf{T}_{m}\right)+\omega_{12}, \\
\dot{\mathbf{Y}}_{P_{1}}=\mathbf{K}^{-1} \cdot \mathbf{F}_{P_{1} P_{2}}+\mathbf{u}_{12} .
\end{gathered}
$$

The initial particle angles $\theta_{01}$ and $\theta_{02}$ can be different. At the first instants, particles align with the magnetic field direction. After that, with the departure from one equilibrium orientation to the other, torques applied on both particles are almost equal, and the angular velocities too. Therefore, one notation $\theta$ will be shortly used to refer to the angle of both particles with the magnetic field direction. In the translation equation of motion, the coefficients of the mobility matrix $\mathbf{K}^{-1}$ depend exclusively on this orientation angle $\theta$. Equations (11) and (12) were solved numerically to obtain the particle trajectories, using the same integration scheme as Sec. II B.

Figure 8 reveals that Eqs. (11) and (12) allow predicting particle angular and translational motion in agreement with numerical simulations. Also particle separation occurs in a 

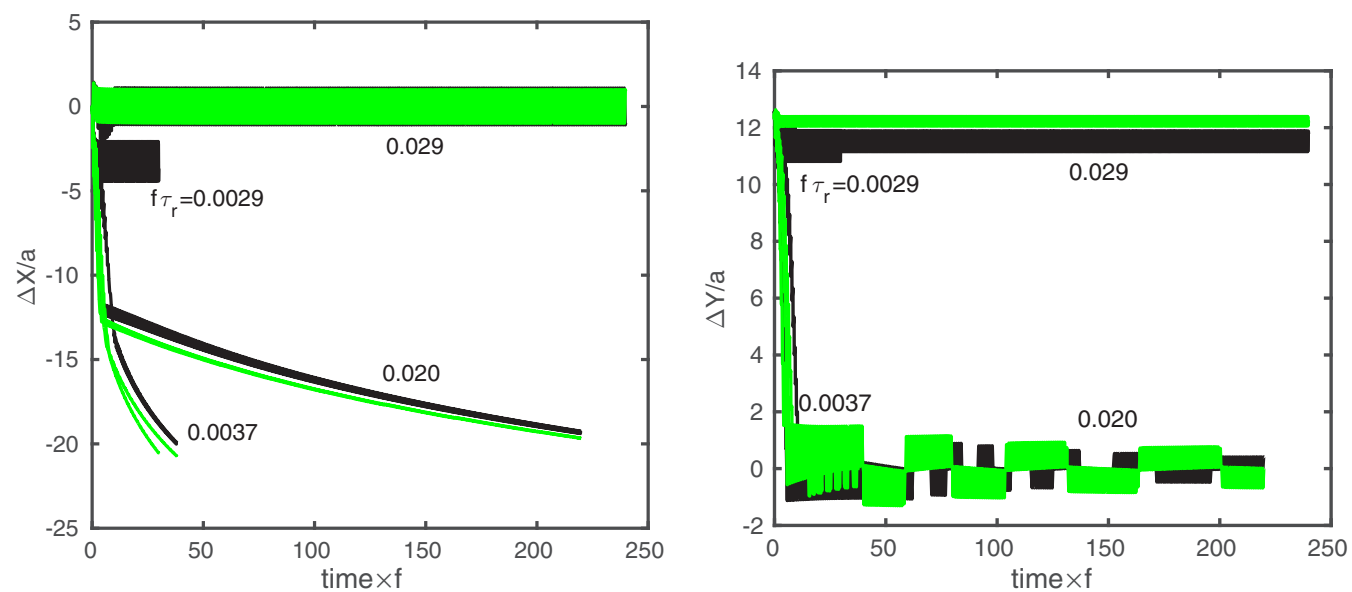

FIG. 8. Idem Fig. 6. Black lines are from FCM simulations. Green lines are predicted using the simplified model [Eqs. (11) and (12)] at identical frequencies.

range of frequencies $\left[f_{c 1}-f_{c 2}\right] \tau_{r}$ close to the range found in numerical simulations $\approx[0.0037-0.029]$. Therefore, we used this model to further understand the role of $\mathrm{HI}$ and frequency in cluster breakup.

\section{Discussion on the mechanism leading to particle separation}

Since the particle separation is intimately related to the hydrodynamic repulsion during their rotation under the alternating field, a particular attention was paid to the particle angular motion during the first periods, which are determinant for the separation. Figure 9 shows the angular trajectories of both particles, revealing that they rotate in a synchronous way when hydrodynamic interactions are taken into account, at least up to the critical frequency $f_{c 2}$. The regime of asynchronous rotation is shifted toward higher frequencies (depending on the initial angular configuration) in the presence of hydrodynamic interactions. The system behavior close to the critical frequencies is examined in this section.

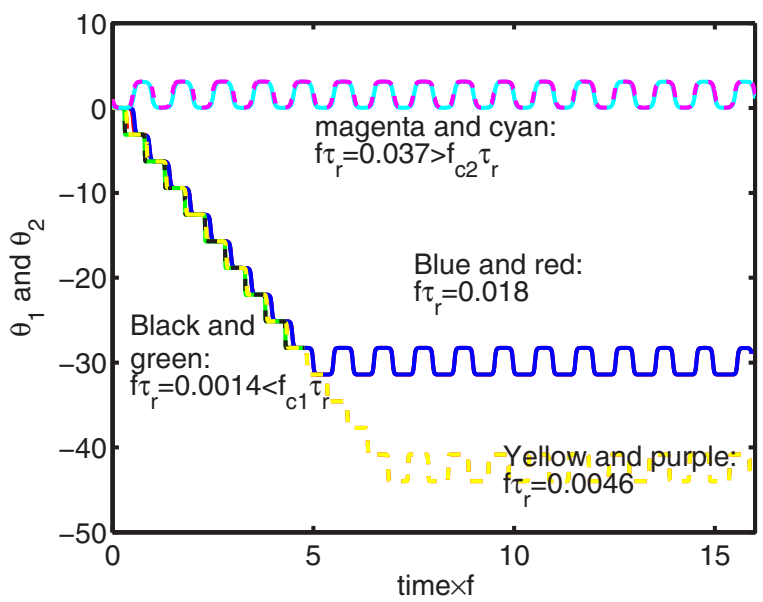

FIG. 9. Angular trajectories of both particles computed while hydrodynamic interactions are taken into account, for $H_{0} / M=1$. Initial angles are $\theta_{10}=\pi / 9$ and $\theta_{20}=\pi / 3$. The initial angle does not have a significant effect in the range of frequency shown here.
Close to the lowest critical frequency $f_{c 1}$, Fig. 10 shows the evolution of configuration $\mathrm{A}$ when $\mathrm{HI}$ are taken into account for two frequencies $f \tau_{r}=0.0014$ and 0.0032 (knowing that $\left.0.0014<f_{c 1} \tau_{r}<0.0032\right)$. Due to the initial departure from the field direction $\left(\theta_{0}=20^{\circ}\right)$, the magnetic torque $T_{m}$ is different from zero at the first instants, and both particles rotate toward the field direction. The plateaus in the curve of $\theta$ correspond to particle quasialignment with the field. The departure from this equilibrium angular position depends on the intensity of the magnetic torque, which should overcome the torque due to interparticle magnetic attraction so that rotation can take place. According to Fig. 10, the maximum torque [proportional to $\sin \theta \cos (\omega t)]$ seems to depend dras-
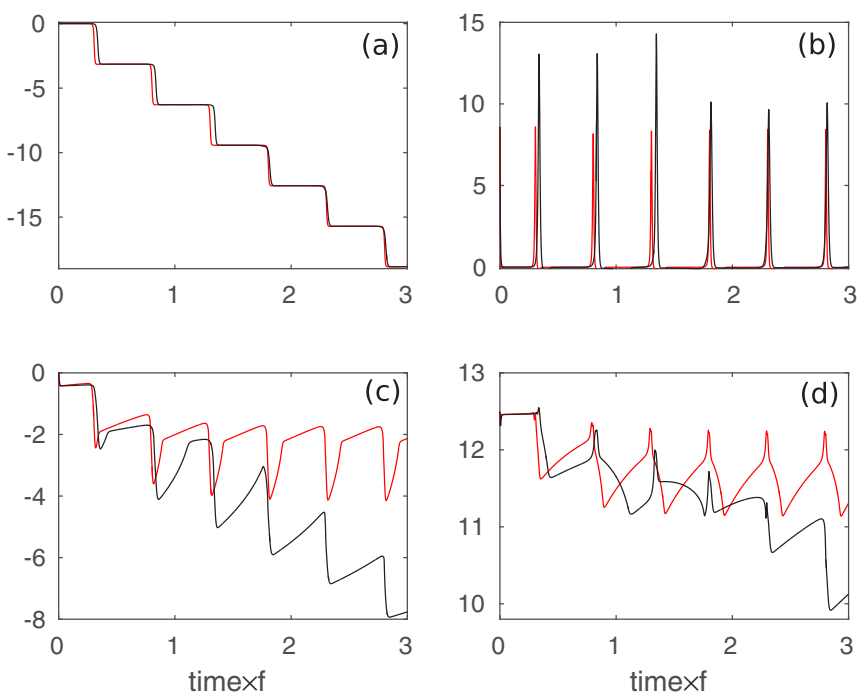

FIG. 10. Difference in the behavior of particle pair for frequencies below and above $f_{c 1}$, starting from configuration $\mathrm{A}$, when $\mathrm{HI}$ are taken into account $\left(f \tau_{r}=0.0014\right.$ with red line and $f \tau_{r}=0.0032$ with black line). A zoom on the first three periods is shown here. The angular trajectory (a) and magnetic torque (b) scaled with $\mu_{0} M^{2} a^{3}$ are plotted for particle $P_{1}$. The relative trajectory of the centers $\Delta X$ (c) and $\Delta Y$ (d) are scaled with $a$. The angle $\theta$ is given in radians. 

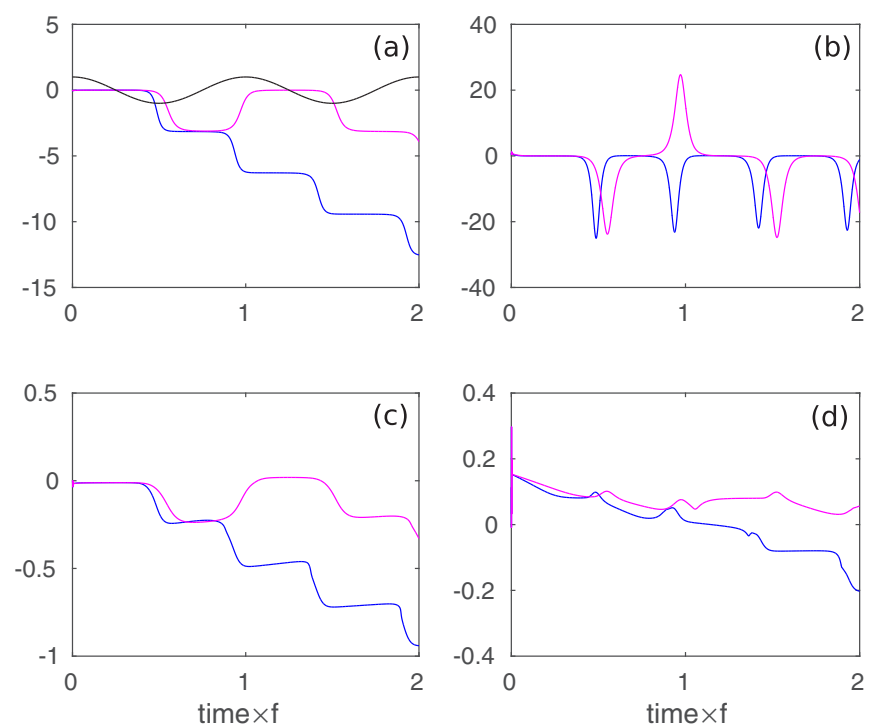

FIG. 11. Idem Fig. 10 with frequencies below and above $f_{c 2}$ (curves in blue are for $f \tau_{r}=0.018$ and in pink for $f \tau_{r}=0.03$ ). The cosine function is added in black in (a) to compare the lag of the particle orientation departure from the field orientation.

tically on the frequency. Additional calculations (not shown here) reveal that the torque increases monotonously with the frequency around $f_{c 1}$. Stronger torques lead to stronger Rotlet perturbation velocity (the Stokeslet one is much smaller in all cases), which acts in favor of particle separation. When the Rotlet component $\mathbf{u}_{R}$ is switched off, fibers in configuration A behave similarly to the case without HI. All these findings do not depend significantly on the initial particle angles $\theta_{10}$ and $\theta_{20}$.

The bifurcation (from separation to nonseparation regime) around $f_{c 2}$ seems to coincide with the transition of the particle rotation from a complete rotation (of $2 \pi$ for every period) toward a back and forth oscillation regime (as it can be seen from Figs. 9 and 11). Actually, the regime of separation seems not to be related to the synchronization of rotation but to the fact that the particles continuously rotate in the same direction. Above the critical frequency $f_{c 2}$ there is a regime of rotation of $\pi$ back and forth, then at higher frequencies the angular amplitude of this oscillation is reduced. As shown in Fig. 12, the first half period generates a lateral displacement; then the separation in the second half period will be more important if they continue to rotate in the same direction than if they rotate back. This regime of oscillation does not induce a separation contrary to the one of continuous rotation. Therefore, the highest transition frequency, $f_{c 2}$, seems to depend on the nature of particle rotation, which is mainly a function of the product $H_{0} M$.

The transition from no-separation to separation around $f_{c 1}$ takes place when the hydrodynamic repulsion due to particle rotation prevails. When the particles abruptly rotate of $\pi$ the average torque is readily obtained by integrating $d \theta / d t$ over half a period, which leads to $\langle T\rangle=\omega \alpha$ that is decreasing function with the frequency. The translational velocity of particle 1 induced by the velocity field coming from the torque on the particle 2 is proportional to $\langle T\rangle$. On the other hand, the

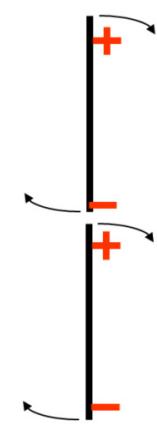

A-initial position

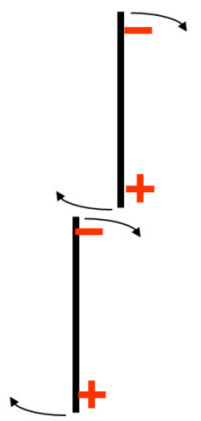

B-Rotation in the same direction as the first one

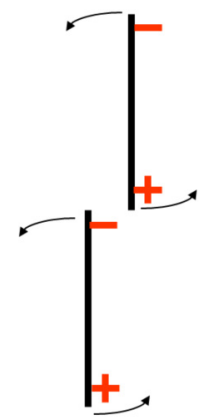

C-Inversion of rotation
FIG. 12. Impact of the rotation direction on the repulsion: A-B are rotating in the same directions. A-C are rotating in opposite directions.

attractive velocity coming from the remnant magnetization, $M$, scales as $M^{2}$. Taking into account the friction coefficient, we obtain the ratio of the hydrodynamic repulsive velocity to the magnetic attractive one $V_{\text {hydro }} / V_{F}=\left(\omega \tau_{r}\right)(\pi \kappa)\left(H_{0} / M\right)$. The coefficient $\kappa$ accounts for the increase of viscous friction on the ellipsoidal particle compared to the spherical one, and its value is between $K_{11}$ and $K_{22}$, which are the diagonal coefficients of the resistance matrix. The critical frequency $f_{c 1}$ can be thought as the frequency at which the balance is obtained between $V_{\text {hydro }}$ and $V_{F}$. Therefore, $\left(f_{c 1} \tau_{r}\right) \propto$ $\left(M / H_{0}\right)$.

\section{E. Effect of $\frac{H_{0}}{M}$}

It was evidenced in the previous sections that the breakup of fiber pair in a head-to-tail configuration depends on the competition between hydrodynamic repulsive force taking place from particle rotation on the one hand and attractive magnetic forces on the other hand. Different tests were realized with $0.05 \leqslant \frac{H_{0}}{M} \leqslant 10$, while the product $H_{0} M$ was kept constant (leading to invariant fiber rotation time scale). The range of frequency $\left[f_{c 1}-f_{c 2}\right]$ leading to particle separation is displayed in Table II for different values of $\frac{H_{0}}{M}$. The upper critical frequency is not significantly dependent on the ratio $\frac{H_{0}}{M}$ if the product $H_{0} M$ is unchanged. Actually, at high frequency the fiber rotation tends toward the vibrating regime, and therefore hydrodynamic repulsion loses efficiency, regardless of the ratio of the magnetic field to the particle magnetization.

TABLE II. Dependence of the range of frequencies that allow particle separation on the ratio of the magnetic field intensity to particle magnetization.

\begin{tabular}{lc}
\hline \hline$\frac{H_{0}}{M}$ & {$\left[f_{c 1}-f_{c 2}\right] \tau_{r}$} \\
\hline 0.05 & {$[0.023-0.037]$} \\
0.1 & {$[0.019-0.037]$} \\
0.5 & {$[0.0046-0.037]$} \\
1 & {$[0.0036-0.029]$} \\
2 & {$[0.0014-0.028]$} \\
10 & {$[0.0004-0.023]$} \\
\hline \hline
\end{tabular}


TABLE III. Range of frequencies allowing particle separation for different particle aspect ratios $\lambda . H_{0} / M=1$ for these tests.

\begin{tabular}{cc}
\hline \hline$\lambda$ & {$\left[f_{c 1}-f_{c 2}\right] \tau_{r}$} \\
\hline 6 & {$[0.0036-0.029]$} \\
5 & {$[0.0032-0.025]$} \\
4 & {$[0.0031-0.025]$} \\
\hline \hline
\end{tabular}

However, the low critical frequency $f_{c 1}$ depends drastically on $\frac{H_{0}}{M}$. We actually observe an increase of $f_{c 1} \tau_{r}$ with $M / H_{0}$ (or a decrease with $H_{0} / M$ ) but less than a simple linear law (as expected from $V_{\text {hydro }} / V_{F}=f_{c 1} \tau_{r} \pi H_{0} / M=1$ ); this discrepancy likely comes from the effect of the translational velocity induced by the Stokeslet that we have neglected in this simplified approach.

\section{F. Effect of the aspect ratio $\lambda$}

All the results shown in this paper were obtained for a particle aspect ratio equal to 6 . The results on particle separation may depend on the aspect ratio in a non trivial way. At low frequencies, the bifurcation near the critical frequency $f_{c 1}$ predicted from $V_{\text {hydro }} / V_{F}=\left(f_{c 1} \tau_{r}\right)\left(2 \pi^{2} \kappa\right)\left(H_{0} / M\right)=1$ suggests that $f_{c 1}$ is inversely proportional to the increase in the friction coefficient when the particle aspect ratio increases. However, this analysis does not take into account the fact that the magnetic forces are stronger during particle rotation when particles are smaller (being closer to each other). As for the bifurcation near $f_{c 2}$, it is not expected to depend significantly on the aspect ratio, since the nature of particle rotation is barely dependent on $\lambda$.

We realized some tests, using both the numerical simulations and the simple model, with decreasing the aspect ratio down to 3 , while the magnetic field strength, particle magnetization and particle minor axis were kept constant. The range of frequencies at which particle separation takes place, for different $\lambda$, is summarized in Table III. This table shows that critical frequencies are almost independent of $\lambda$, when the frequency is scaled by the inverse of the particle rotation rate $\tau_{r}$. Note that the rotation time scale increases almost linearly with the aspect ratio.

\section{CONCLUSION}

Numerical simulations and theoretical modeling were used to analyze the motion of two elongated spheroidal particles under the action of an externally imposed alternating magnetic field. The case of ferromagnetic particles in suspension in a viscous fluid was considered. Fibers were submitted to a magnetic torque that leads them to rotate in order to align with the magnetic field direction and to magnetic forces that are mainly attractive when particles are initially in a headto-tail configuration. During particle rotation, hydrodynamic repulsive forces take place from the torque-velocity coupling due to particle anisotropic shape.

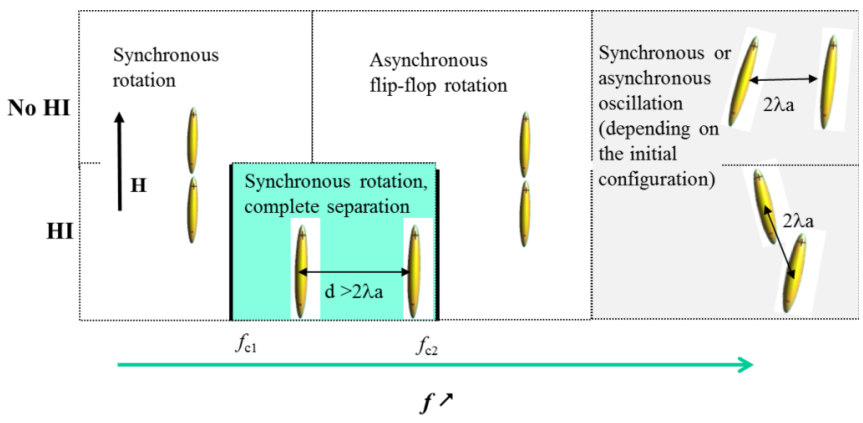

FIG. 13. Scheme summarizing the effect of the frequency on the dynamics of a particle pair, initially placed in a head-to-tail configuration. The final configuration is sketched in each situation, according to the most probable scenario.

The dynamics of a particle pair under an alternating field is sketched qualitatively as a function of the field frequency in Fig. 13. In the absence of hydrodynamic interactions, three different rotational dynamics can be identified: a synchronous rotation at low frequency, an asynchronous flip-flop motion in some intermediate range, and an oscillation above a given frequency. The limit of these three domains depends strongly on the coupling with the translational velocities. In the third domain, at the highest frequencies, we observe that the fiber positions switch from head-to-tail to side-by-side, keeping however constant the distance between the centers. Introducing hydrodynamic interactions between fibers shifts the asynchronous flip-flop regime toward higher frequencies and deeply changes the correlation between the orientational dynamics and the separation regime: with HI, separation occurs in the regime of synchronous rotation $\left(f<f_{c 2}\right)$ at least if the frequency is high enough $\left(f>f_{c 1}\right)$ for the hydrodynamic interactions (proportional to $H$ ) to dominate the attractive one (proportional to $M$ ). For $f_{c 1}<f<f_{c 2}$, separation keeps growing when the particles have attained their position side by side. No separation occurs in the oscillation regime $\left(f>f_{c 2}\right)$ in which case the dynamics is similar with or without HI.

The conclusions of this study were obtained with a pair of ellipsoidal particles having an aspect ratio equal to 6 . The range of frequencies that allowed particle separation were shown to be independent of the aspect ratio if the frequency of the alternating magnetic field was scaled by the inverse of the particle rotation time scale (which itself depends on the aspect ratio). Systems that consist of a bulk of ellipsoidal particles with random initial orientation with respect to the magnetic field will be definitely an interesting subject for future investigation.

\section{ACKNOWLEDGMENTS}

The authors acknowledge E. Keaveny for useful discussion on modeling of hydrodynamic perturbations and B. Delmotte for the basic version of the FCM numerical code. This work was granted access to the HPC resources of CALMIP under the allocation 2015 and 2016-P1002 and of GENCI under the allocations x20132a6942. 
[1] D. Klingenberg, AIChE J. 47, 246 (2001).

[2] M. T. López-López, P. Kuzhir, and G. Bossis, J. Rheol. 53, 115 (2009).

[3] P. Kuzhir, M. T. López-López, and G. Bossis, J. Rheol. 53, 127 (2009).

[4] L. M. Armijo, Y. I. Brandt, D. Mathew, S. Yadav, S. Maestas, A. C. Rivera, N. C. Cook, N. J. Withers, G. A. Smolyakov, N. L. Adolphi, T. C. Monson, D. L. Huber, H. D. C. Smyth, and M. Osiński, Nanomaterials 2, 134 (2012).

[5] N. Pera, A. Kouki, S. Haataja, H. Branderhorst, R. Liskamp, G. Visser, J. Finne, and R. Pieters, Org. Biomol. Chem. 8, 2425 (2010).

[6] A. Orban, A. Butykai, A. Molnar, Z. Prohle, G. Fulop, T. Zelles, W. Forsyth, D. Hil, I. Muller, L. Schofield, M. Rebelo, T. Hanscheid, S. Karl, and I. Kezsmarki, PLoS ONE 9, e96981 (2014).

[7] P. Tierno, Phys. Chem. Chem. Phys. 16, 23515 (2014).

[8] T. Waigh, Rep. Prog. Phys. 79, 074601 (2016).

[9] A. Brasovs, E. K. Cimurs, J. A. Zeltins, J. Berret, and A. Cebers, Soft Matter 11, 2563 (2015).

[10] R. Dreyfus, J. Baudry, M. Ropper, M. Fermigier, H. Stone, and J. Bibette, Nature 437, 862 (2005).
[11] E. Keaveny and M. Maxey, J. Fluid Mech. 598, 293 (2008).

[12] F. Martinez-Pedrero, A. Cebers, and P. Tierno, Soft Matter 12, 3688 (2016).

[13] T. Kang, Y. Gao, M. Hulsen, J. den Toonder, and P. Anderson, Comput. Fluids 86, 569 (2013).

[14] D. Klingenberg, F. Van Swol, and C. Zukoski, J. Chem. Phys. 91, 7888 (1989).

[15] J. Happel and H. Brenner, Low Reynolds Number Hydrodynamics (Martinus Nijhoff, Boston,1986).

[16] D. Liu, E. Keaveny, M. Maxey, and G. Karniadakis, J. Comput. Phys. 228, 3559 (2009).

[17] G. Bossis, O. Volkova, S. Lacis, and A. Meunier, Lect. Notes Phys. 594, 202 (2003).

[18] S. Melle, O. G. Calderon, M. A. Rubio, and G. G. Fuller, Phys. Rev. E 68, 041503 (2003).

[19] M. Belovs and A. Cebers, J. Phys. A: Math. Theor. 44, 295101 (2011).

[20] M. Maxey and B. Patel, Int. J. Multiphase Flow 27, 1603 (2001).

[21] S. Lomholt and M. Maxey, J. Comput. Phys. 184, 381 (2003).

[22] E. Guazzelli and J. Morris, A Physical Introduction to Suspension Dynamics (Cambridge University Press, Cambridge, 2012).

[23] G. K. Batchelor, J. Fluid Mech. 44, 419 (1970). 